\title{
1 A novel metabarcoded DNA sequencing tool for the detection of 2 Plasmodium species in malaria positive patients.
}

3

4 Abdul Wahab ${ }^{\mathrm{a} 1}$, Ayaz Shaukat ${ }^{\mathrm{bl}}$, Qasim Ali ${ }^{\mathrm{cl}}$, Mubashir Hussain ${ }^{\mathrm{a}}$, Taj Ali Khan ${ }^{\mathrm{a}}$, M. Azmat

5 Ullah Khan $^{\mathrm{b}}$, Imran Rashid ${ }^{\mathrm{d}}$, Mushtaq A. Saleem ${ }^{\mathrm{b}}$, Mike Evans ${ }^{\mathrm{e}}$, Neil D. Sargison ${ }^{\mathrm{e} *}$, Umer

6 Chaudhry ${ }^{\mathrm{f}} *$

7

$8{ }^{a}$ University of Science and Technology, Kohat, Khyber Pakhtunkhwa, Pakistan

$9{ }^{b}$ University of Central Punjab, Lahore, Punjab, Pakistan

${ }^{c}$ Gomal University, Dera Ismail Khan, Khyber Pakhtunkhwa, Pakistan

${ }^{d}$ University of Veterinary and Animal Sciences, Lahore, Punjab, Pakistan

${ }^{e}$ Royal Dick School of Veterinary Studies, University of Edinburgh, UK

${ }^{f}$ Roslin Institute, University of Edinburgh, UK

1 Contributed equally

*Corresponding author:

Umer Chaudhry

Email: uchaudhr@exseed.ed.ac.uk,Tel: 00441316519244

20

University of Edinburgh, The Roslin Institute, Easter Bush Veterinary Centre, UK, EH25 9RG

Neil Sargison

Neil.Sargison@ed.ac.uk, +44 (0)131 6517300

University of Edinburgh, R(D)SVS, Easter Bush Veterinary Centre, UK, EH25 9RG 


\section{Abstract}

Various PCR based methods have been described for the diagnosis of malaria, but most depend on the use of Plasmodium species-specific probes and primers; hence only the tested species are identified and there is limited available data on the true circulating species diversity. Sensitive diagnostic tools and platforms for their use are needed to detect Plasmodium species in both clinical cases and asymptomatic infections that contribute to disease transmission. We have been recently developed for the first time a novel high throughput 'haemoprotobiome' metabarcoded DNA sequencing method and applied it for the quantification of haemoprotozoan parasites (Theleria and Babesia) of livestock. Here, we describe a novel, high throughput method using an Illumina MiSeq platform to demonstrate the proportions of Plasmodium species in metabarcoded DNA samples derived from human malaria patients. Plasmodium falciparum and Plasmodium vivax positive control gDNA was used to prepare mock DNA pools of parasites to evaluate the detection threshold of the assay for each of the two species and to assess the accuracy of proportional quantification. We then applied the assay to malaria-positive human samples to show the species composition of Plasmodium communities in the Punjab province of Pakistan and in the Afghanistan-Pakistan tribal areas. The diagnostic performance of the deep amplicon sequencing method was compared to an immunochromatographic assay that is widely used in the region. Metabarcoded DNA sequencing showed better diagnostic performance, greatly increasing the estimated prevalence of Plasmodium infection. The next-generation sequencing method using metabarcoded DNA has potential applications in the diagnosis, surveillance, treatment, and control of Plasmodium infections, as well as to study the parasite biology.

Keywords: Malaria, Plasmodium falciparum and Plasmodium vivax, metabarcoded DNA, deep amplicon sequencing.

60 


\section{Introduction}

Malaria is the most important vector-borne disease, causing high morbidity and mortality (Moody, 2002). More than 3.4 billion people are infected, resulting in an estimated 1.2 billion malaria cases every year (Poostchi et al., 2018). The disease is caused by intracellular protozoan parasites of the genus Plasmodium transmitted through Anopheles mosquitoes (Dash et al., 2007; Sinka et al., 2012). Plasmodium has an indirect life cycle including one stage in Anopheles mosquitoes and three different stages in humans, all with different rates of replication ( $\mathrm{Li}$ et al., 1997). Gametogenesis occurs in human blood and fertilisation of male and female macro and microgametes occurs in the midgut of the mosquito after feeding. Asexual stages occur in the gut of the mosquito as sporogony, and after biting the humans, sporozoites undergo exoerythrocytic schizogeny in the hepatic cells and then erythrocytic schizogony in the blood cells ( $\mathrm{Li}$ et al., 1994b). Five species of Plasmodium parasite infect humans, namely Plasmodium falciparum, Plasmodium vivax, Plasmodium ovale, Plasmodium malariae and Plasmodium knowlesi. P. falciparum is the most associated with lethal disease, but in recent years, there has been an increase in disease severity attributable to $P$. vivax (Saravu et al., 2014; Scholzen et al., 2014; William et al., 2011).

The 18S rDNA of Plasmodium is unique due to its genomic arrangement dispersed among different chromosomes. The copy number is limited to 4 to 8 per genome and the sequences are not identical ( $\mathrm{Li}$ et al., 1994b). Their expression is regulated during different development stages of the life cycle ( $\mathrm{Li}$ et al., 2014); for example, at least three types of genotypic variants have been identified between stages of $P$. falciparum and $P$. vivax laboratory isolates ( $\mathrm{Li}$ et al., 1994b; McCutchan et al., 1988; Qari et al., 1994; Rogers et al., 1995). However, the presence of these variants has not been reported in the field studies.

The 18S rDNA of Plasmodium forms a mosaic of conserved and variable regions; whereby the conserved regions contribute to form a secondary structure of rRNA that appears to be associated with the universal function of the ribosomes. The variable regions are scattered among the conserved regions and contribute to major differences in gene composition and size ( $\mathrm{Li}$ et al., 1994a). The function of variable regions is not fully understood, but determination of sequence variations can discriminate between Plasmodium species (Agudelo et al., 2013; Haanshuus et al., 2013; Lee et al., 2015; Lefterova et al., 2015), and overcome limitations of traditional microscopic and immunochromatographic methods for the diagnosis of this group of parasites at species level. 
Significant progress has been made in the global fight against malaria through high throughput rapid diagnosis. Sensitive diagnostic tools are needed to detect clinically and subclinically infected patients (Echeverry et al., 2016). Molecular methods including qPCR, species-specific PCR, nested PCR, and multiplex PCR have been described (Canier et al., 2013; Cunha et al., 2009; Das et al., 1995; Echeverry et al., 2016; Haanshuus et al., 2013; Steenkeste et al., 2009), but these are low throughput, hence relatively expensive (Chaudhry et al., 2019). These methods depend on the use of species-specific probes and primers, meaning that only the tested species are identified, hence are limited in their ability to describe true circulating species diversity (Moody, 2002). In contrast, high throughput metabarcoded DNA sequencing using the Illumina MiSeq platform is relatively low-cost and potentially less error-prone. We have applied this 'haemprotobiome' method, to the study of tick-borne haemoprotozoan parasites of ruminants (Chaudhry et al., 2019). The method has the potential to open new areas of research in the study of Plasmodium, to accurately provide relative quantification of co-infecting species and to evaluate drug treatment responses (Shaukat et al., 2019). The method uses primers binding to the conserved sites and analyse of up to $600 \mathrm{bp}$ sequence reads. The use of adapter and barcoded primers allows a large number of samples to be pooled and sequenced in a single MiSeq flow cell, making the assay suitable for high-throughput analysis (Shaukat et al., 2019).

Here, we report for the first time the development of a deep sequencing method using the Illumina MiSeq platform to quantify $P$. falciparum and $P$. vivax present in malaria-positive human blood samples. The results are compared with a standard immunochromatographic assay to validate the method's accuracy for species identification.

\section{Materials and methods}

\subsection{Parasite material}

Positive control samples of $P$. falciparum were kindly provided by D Jason Mooney at the Roslin Institute, University of Edinburgh, UK, previously obtained from National Institute for Biological Standards and Control (NIBSC code 04/176). P. vivax control samples were kindly provided by Dr Imran Rashid at the University of Veterinary and Animal Sciences, Lahore, Pakistan, previously obtained from the Biodefense and Emerging Infections Research Resources Repository (BEI code MRA-41). Four replicates each of mock pools comprising of P. falciparum only (Mix 1), P. vivax only (Mix 2), and P. falciparum and P. vivax (Mix 3) were created. These were used to test the detection threshold of the metabarcoded sequencing 
132

133

134

135

136

137

138

139

140

141

142

143

144

145

146

147

method and to show the proportions of each of the Plasmodium species present. Four negative control of human blood samples were provided by Sana Amir and Saqib Shahzad, Chughtai Diagnostic Laboratory, Lahore Pakistan.

Malaria suspected patients referred to Basic Health Units in the tribal areas of the Afghanistan-Pakistan border and Chughtai Diagnostic Laboratory in the Punjab province of Pakistan were invited to participate in this study. Prior discussions were held with key administrative and community leaders to raise awareness of the study. Samples were taken by trained para-medical workers under the supervision of local collaborators and the Basic Health Unit or Chughtai Diagnostic Laboratory staff. The institutional review boards of the University of Central Punjab (UCP-30818), and the Kohat University of Science and Technology, Pakistan (KUST/EC/1379) approved the study. Patients of all age groups were included in this study with symptoms consistent with malaria, including vomiting, fever, headache, chills, sweats, nausea and fatigue.

Blood samples were collected by venipuncture during peak malaria transmission seasons between August to November 2017 and 2018. A total of $5 \mathrm{ml}$ of intravenous blood was drawn into EDTA tubes and stored at $-20{ }^{0} \mathrm{C}$ for gDNA isolation. Each sample was also routinely analysed by microscopic examination under oil immersion (x1000) of 4\% Giemsa-stained blood smears for the diagnosis of malaria. The Plasmodium goes through different stages of their development cycle (48 hr), which gives the parasites a different visual appearance that can be observed under the microscope. These stages show the ring (Fig. 1A), tropnozoite, schizont, and gametocyte appearance. Malaria case identification was based on the appearance of those stages on the microscopic examination (Fig. 1A). Overall, 365 malaria suspected positive patients were identified.

\subsection{Immunochromatographic assay}

The 365 malaria-positive on microscopic examination blood samples, each having an unknown level of parasitemia, were analysed using a commercial immunochromatographic rapid diagnostic test (RDT) kit. The Malaria Pf/Pv Ag Rapid Test (Healgen ${ }^{\circledR}$; Zhejiang Orient Gene Biotech Co, Ltd) RDT kit was designed to detect $P$. falciparum-specific histidine-rich protein 2 (Pf-HRP2) and P.vivax-specific lactate dehydrogenase (Pv-LDH). The kit was transported and maintained at the room temperature, opened just before use to avoid humidity damage, and used in accordance with the manufacturer's recommendations. During the assay, an adequate volume of the blood sample was dispensed into the sample well ' $S$ ' of the test cassette and the lysis buffer is added to the buffer well ' $\mathrm{B}$ '. The buffer contains a detergent 
166 that lyses the red blood cells and releases antigens, which migrate by capillary action across

167

168

169

170

171

172

173

174

175

176

177

178

179

180

181

182

183

184

185

186

187

188

189

190

191

192

193

194

195

196

197

198

199 the strip held in the cassette. If Pf-HRP2 binds to the HRP2 gold conjugates and the immunocomplex is then captured on the membrane by the pre-coated anti-Pf-HRP2 antibodies, forming a burgundy colored pf band, indicating $P$. falciparum positive test (Fig. 1B). If Pv-LDH binds to the LDH gold conjugates and the immunocomplex is then captured on the membrane by the pre-coated anti-Pv-LDH antibodies, forming a burgundy colored pv band, indicating $P$. vivax positive test (Fig. 1B). The absence of any band suggests a negative result. The test also contained an internal control ' $\mathrm{C}$ ' band, exhibiting a burgundy colored band of the immunocomplex of goat anti-mouse IgG/mouse IgG (anti-Pv-LDH and anti-PfHRP2) gold conjugates, regardless of the color development on ' $\mathrm{C}$ ' band.

\subsection{Genomic DNA isolation, primer design and adapter/barcoded PCR amplification of rDNA 185 locus}

$50 \mu \mathrm{l}$ of blood from each of the 365 samples was used as a template, to extract gDNA according to the protocols described in the TIANamp blood DNA kit (Tiangen Biotech (Beijing) Co., Ltd). Overall, $589 \mathrm{bp}$ and $568 \mathrm{bp}$ fragments of $P$. falciparum and $P$. vivax $18 \mathrm{~S}$ rDNA, respectively, were amplified using newly developed adapter primer sets (Supplementary Table S1). The overall scheme of the sample preparation is described in Figure 2A. Adapters were added to these primers to allow the successive annealing of subsequent metabarcode primers and $\mathrm{N}$ is the number of random nucleotides included between the locus-specific primers and adapter to increase the variety of generated amplicons as previously described by Chaudhry et al. (2019). Four forward (Plasmo1_For, Plasmo1_For-1N, Plasmo1_For-2N, Plasmo1_For-3N) and four reverse (Plasmo2_Rev, Plasmo1_Rev-1N, Plasmo1_Rev-2N, Plasmo1_Rev-3N) primers were mixed in equal proportion (Supplementary Table S1) and used for first-round PCR under the following conditions: 5X KAPA buffer, 10mM dNTPs, $10 \mathrm{uM}$ forward and reverse adapter primer, 0.5 U KAPA Polymerase (KAPA Biosystems, USA), and $1 \mathrm{ul}$ of worm lysate. The thermocycling conditions of the PCR were $95^{\circ} \mathrm{C}$ for $2 \mathrm{~min}$, followed by 35 cycles of $98^{\circ} \mathrm{C}$ for $20 \mathrm{sec}, 60^{\circ} \mathrm{C}$ for $15 \mathrm{sec}, 72^{\circ} \mathrm{C}$ for $15 \mathrm{sec}$ and a final extension $72^{\circ} \mathrm{C}$ for $5 \mathrm{~min}$. PCR products were purified with AMPure XP Magnetic Beads (1X) (Beckman Coulter, Inc.).

After the purification, a second-round PCR was performed by using sixteen forward and twenty-four reverse barcoded primers. The barcoded forward (N501 to N516) and reverse (N701 to N724) primers (10 uM each) were previously described by Chaudhry et al. (2019). The primers were used in a manner that repetition of same forward and reverse sequences did 
not occur in the different samples. The second-round PCR conditions were: 5X KAPA buffer, $10 \mathrm{mM}$ dNTPs $0.5 \mathrm{U}$ KAPA Polymerase (KAPA Biosystems, USA), and $2 \mathrm{ul}$ of first-round PCR product as DNA template. The thermocycling conditions of the second round PCR were $98^{\circ} \mathrm{C}$ for $45 \mathrm{sec}$, followed by 7 cycles of $98^{\circ} \mathrm{C}$ for $20 \mathrm{sec}, 63^{\circ} \mathrm{C}$ for $20 \mathrm{sec}$, and $72^{\circ} \mathrm{C}$ for 2 minutes. PCR products were purified with AMPure XP Magnetic Beads (1X) according to the protocols described by Beckman Coulter, Inc.

\subsection{Sequencing of metabarcoded $18 S$ rDNA, data handling, and bioinformatic analysis}

The pooled library was measured with KAPA qPCR library quantification kit (KAPA Biosystems, USA). The prepared library was then run on Illumina MiSeq Sequencer using a 600-cycle pair-end reagent kit (MiSeq Reagent Kits v2, MS-103-2003) at a concentration of $15 \mathrm{nM}$ with an addition of 25\% Phix Control v3 (Illumina, FC-11-2003).

The overall scheme of the data handling and bioinformatics analysis is described in Figure 2B. MiSeq data were handled with our own bioinformatics pipeline (Chaudhry et al., 2019). Briefly, MiSeq separates all sequence reads during post-run processing using the barcoded indices and to generate FASTQ files. The raw paired read-ends were run into the 'make.contigs' command to combine the two sets of reads for each sample. The command extracts sequence and quality score data from the FASTQ files, creating the complement of the reverse and forward reads, and then joining the reads into contigs. After removing the too long, or ambiguous sequence reads, the data were then aligned with the $P$. falciparum and $P$. vivax reference sequence library (for more details Supplementary Data S1 and Result section 3.1) using the 'align.seqs' command to summarise the 589 bp and 568 bp fragments encompassing parts of the 18S DNA spanning the hyper-variable region of the $P$. falciparum and $P$. vivax ribosomal cistrons. At this stage, the $18 \mathrm{~S}$ rDNA analysis was completed by classifying the sequences into either of the two species by using the 'classify.seqs' command and creating a taxonomy file by using the 'summary.tax' command. Overall, 762674 million reads of $18 \mathrm{~S}$ rDNA were generated from the data set.

For the phylogenetic analysis of $P$. falciparum and $P$. vivax $18 \mathrm{~S}$ rDNA, all the classified sequences were run on the 'screen.seqs' command and the count list of the consensus sequences of each sample was created using the 'unique.seqs' command followed by the use of the 'pre.cluster' command to look for sequences differences and to merge them in groups based on their abundance. Any chimeras were identified and removed by using the 'chimera.vsearch' command. The count list was further used to create the FASTQ files of the 
233 consensus sequences of each sample using the 'split.groups' command (for more details

234 Supplementary Data S2).

235

2.5. Split and maximum-likelihood trees of P. falciparum and $\mathrm{P}$. vivax $18 S$ rDNA sequences

$P$. falciparum and $P$. vivax $18 \mathrm{~S}$ rDNA sequence reads were analysed separately in Geneious v9.0.1 software (Kearse et al., 2012) using the MUSCLE alignment tool. The aligned sequences were then imported into the FaBox 1.5 online tool to collapse those with $100 \%$ base pair similarity after corrections into single genotypes. The split tree of $P$. falciparum and $P$. vivax $18 \mathrm{~S}$ rDNA was created in the SplitTrees4 software by using the UPGMA method in the Jukes-Cantor model of substitution (Huson and Bryant, 2005). The maximum-likelihood tree for $P$. falciparum and $P$. vivax $18 \mathrm{~S}$ rDNA was constructed by the HKY model of substitution in the MEGA 7 software and to select the appropriate model of nucleotide substitutions (Tamura et al., 2013).

\subsection{Statistical analysis}

The data were analysed using CompareTests: Correct for Verification Bias in Diagnostic Accuracy and Agreement Statistics software (R package version 1.2.). The frequency of $P$. falciparum and $P$. vivax in the samples was calculated by dividing the number of sequence reads for each sample by the total number of reads. The effect of the mock pools of $P$. falciparum and $P$. vivax positive controls were analysed by running a Kruskal-Wallis ranksum test for each admixture. The performance of the immunochromatographic assay was compared against metabarcoded sequencing, using a Fisher's Exact test to calculate the predictive value and Category-Specific Classification Probability (CSCP) with 95\% confidence interval. Kappa (k) values were calculated to express the agreement beyond chance; where values greater than 0.80 were considered to represent perfect agreement; values of $0.61-0.80$ to represent good agreement; and values of $0.21-0.60$ to represent moderate agreement.

260

\section{Results}

262

\section{1. $P$. falciparum and $P$. vivax $18 S$ rDNA reference sequence libraries}

The sequence reads generated by metabarcoded 18S rDNA sequencing of the $P$. falciparum and $P$. vivax positive controls were compared to the malaria-positive samples and to the published NCBI GenBank 18S rDNA sequences to account for any genetic diversity. A 
267

268

269

270

271

272

273

274

275

276

277

278

279

280

281

282

283

284

285

286

287

288

289

290

291

292

293

294

295

296

297

298

299

300

total of $34 P$. falciparum and $74 P$. vivax reference sequences were identified (Fig. 3, Supplementary Data S1). The maximum-likelihood tree shows distinct clustering of the $P$. falciparum and P. vivax 18S rDNA regions (Fig. 3), hence these closely related Plasmodium species can be reliably differentiated by virtue of $18 \mathrm{~S}$ rDNA sequence variations.

\subsection{Validation of the metabarcoded sequencing assay using mock pools of P. falciparum and} P. vivax

Four replicates each of $P$. falciparum only (Mix 1), P. vivax only (Mix 2), and $P$. falciparum and $P$. vivax (Mix 3) were created from gDNA to demonstrate the detection accuracy of the metabarcoded DNA sequencing method and to show the proportions of each of the species being present (Fig. 4; Supplementary Table S2). The mixing of different mock pools demonstrates the accurate detection ability of the metabarcoded sequencing method and to show the proportions of each of the species being present. The Mix 1 pool yielded only $P$. falciparum sequence reads and the Mix 2 pool yielded only $P$. vivax sequence reads (Fig. 4). The Mix 3 pool yielded both $P$. falciparum and $P$. vivax sequence reads, with no statistically significant variations between replicates (Kruskal-Wallis rank-sum test; Mix1: $\chi 2$ (1) 0, p=1; Mix2: $\chi 2(1)$ 0, p=1; Mix3: $\chi 2(3) 0.02153, p=0.5231)$.

\subsection{Assessment of the immunochromatographic assay and metabarcoded sequencing for the} identification of P. falciparum and P. vivax

The immunochromatographic assay and metabarcoded DNA sequencing methods were applied to malaria-positive blood samples to detect $P$. falciparum and $P$. vivax in the field (Fig. 5, Supplementary Table S3). The results of both assays demonstrate that the prevalence of $P$. vivax infection was higher than that of $P$. falciparum infection. In the case of the metabarcoded DNA sequencing assay, those samples yielding more than 1000 reads (implying sufficient gDNA for accurate amplification) were included in the analysis (Supplementary Table S3). Plasmodium vivax was present in 199 (69.8\%) patients, $P$. falciparum in 84 (29.5\%) and mixed infection in $2(0.7 \%)$ patients (Fig. 5). The immunochromatographic assay showed that Plasmodium vivax was present in 187 (65.6\%) patients, P. falciparum in 78 (27.4\%), mixed infection in $2(0.7 \%)$ patients (Fig. 5) and 18 $(6.32 \%)$ malaria-positive cases were negative in RDT, but positive in the metabarcoded DNA sequencing assay (Fig. 5).

The degree of agreement between the immunochromatographic and metabarcoded DNA sequencing assays was high with $\kappa=0.893$ (95\% CI: 0.839-0.930). The Category-Specific 
301 Classification Probability (CSCP) was also high in all four categories, ranging from 0.892

302

303

304

305

306

307

308

309

310

311

312

313

314

315

316

317

318

319

320

321

322

323

324

325

326

327

328

329

330

331

332

333

334

(95\% CI: 0.807 - 0.943) to 1. The Predictive Values (PV) for 'positive RDT' results were also very high, ranging from 0.962 (0.860 - 0.990) to 1 . However, the PVs for 'negative RDT' results were $0.816(0.724-0.883)$; being significantly lower than the Predictive Value for both 'RDT P. falciparum' ( $\mathrm{p}=0.004)$ and 'RDT $P$. vivax' $(\mathrm{p}<0.001)$ results. In samples with similar disease prevalence, there is, therefore, an increased likelihood that a negative RDT result may be incorrect.

\subsection{Phylogenetic analysis of the P. falciparum and P. vivax rDNA $18 S$ sequences}

Overall, 112 different genotypes of the P. falciparum 18S rDNA locus were identified among 84 field samples and 12 from the NCBI GenBank sequences (Supplementary Data S2 and section 3.1. and 3.3). The split tree shows at least two distinct clades (Fig. 6A), and it sets apart that 9 genotypes in the Clade I, belongs to a 'type S' $18 \mathrm{~S}$ rDNA region (McCutchan et al., 1988). The remaining 115 genotypes were in clade II, belonging to a 'type A' $18 \mathrm{~S}$ rDNA region (McCutchan et al., 1988). 80 genotypes predominated in group 1 and 35 genotypes in group 2 (Fig. 6A). In contrast, 30 different genotypes of the P. vivax 18S rDNA locus was identified among 199 field samples and 18 from the NCBI GenBank sequences (Supplementary Data S2 and section 3.1. and 3.3). The split tree shows at least two distinct clades (Fig. 6B), and it sets apart that 3 genotypes in the Clade I, belong to the 'type S' $18 \mathrm{~S}$ rDNA as described by $\mathrm{Li}$ et al. (1994). The remaining 45 genotypes were in clade II, belonging to 'type A' $18 \mathrm{~S}$ rDNA (Li et al., 1994b). 7 genotypes predominated in groups 1 and 2 , and 31 genotypes in group 3 (Fig. 6B).

\section{Discussion}

Microscopic examination of blood smears remains the mainstay for the diagnosis of Plasmodium in the field. The procedure allows the detection of levels of at least 200 parasites/ul, which is sufficient for the diagnosis of most symptomatic cases, but can result in misdiagnoses at low levels of parasitemia (Rakotonirina et al., 2008; Wongsrichanalai et al., 2007). The method is labor-intensive, time-consuming, and interpretation of results requires highly skilled microscopists (Canier et al., 2013; Echeverry et al., 2016).

The immunochromatographic assay is also utilised in case investigation and malaria surveillance programs in the field. The method depends on the incorporation of conjugated monoclonal antibodies providing the indicator of infection (Moody, 2002; Wongsrichanalai et al., 2007). The targeted antigens are abundant in the sexual and asexual stages of the parasites, 
335 while histidine-rich protein 2 (Pf-HRP2) based RDT is specific for P. falciparum and lactate

336

337

338

339

340

341

342

343

344

345

346

347

348

349

350

351

352

353

354

355

356

357

358

359

360

361

362

363

364

365

366

367

dehydrogenase (Pf-LDH \& Pv-LDH) specific for detecting both $P$. falciparum and $P$. vivax.

Pan based RDT targets specific antigens including lactate dehydrogenase (P-LDH) and aldolase proteins found in all Plasmodium species (Akinyi Okoth et al., 2015; Murillo Solano et al., 2015). Several factors potentially affect the accuracy and false-negative results of RDT including the interpretation of the test strip colour change, the density of malaria infection in the host, improper storage/handling of the kit and poor test performance (Echeverry et al., 2016). Besides this, other major factors such cross-reactivity of HRP2 with histidine-rich protein 3 (a structural homolog with significant sequence similarity) and deletions in the HRP2 locus in P. falciparum isolates may account for false-negative results (Akinyi Okoth et al., 2015; Rachid Viana et al., 2017).

Conventional PCR based molecular methods are useful in the detection of Plasmodium species for which the reagents and conditions have been developed, but have limitations in terms of lacking scalability (Canier et al., 2013; Cunha et al., 2009; Das et al., 1995; Echeverry et al., 2016; Haanshuus et al., 2013; Steenkeste et al., 2009). The diagnostic challenges of the disease identification have not been resolved yet (Wongsrichanalai et al., 2007), therefore the metabarcod DNA sequencing potentially provides a more accurate and reliable automated high-throughput method to detect Plasmodium species in blood samples. The use of a single PCR utilising primers conserved between Plasmodium species provides a powerful tool to measure the relative sequence representation of each species in the blood samples. In the present study, we have evaluated a metabarcoded DNA sequencing method to identify the presence of $P$. falciparum and $P$. vivax using mock parasite pools, applying the method to malaria-positive blood samples, and the detection P. falciparum and P. vivax rDNA18S genotypic variants in the field samples.

We tested the ability of the metabarcoded DNA sequencing assay to accurately determine the relative species proportions in combinations of $P$. falciparum and $P$. vivax. To do this, we generated mock pools containing different estimated proportions of both species and demonstrated no significant variations between replicates. A previous study using pools of laboratory-maintained Theileria and Babesia haemoprotozoan parasites showed that the relative sequence representation was unaffected by either the number of PCR cycles employed or the parasite species composition of the sample. This study also found no sequence representation bias in PCR products used for sequencing, arising from the number of first-round PCR cycles (Chaudhry et al., 2019). 
After validating the metabarcoded DNA sequencing assay using mock pools of Plasmodium positive DNA, we applied the method to field samples collected from suspected malaria-positive patients in the tribal area of the Afghanistan-Pakistan border and in the Punjab province of Pakistan, where malaria caused by $P$. falciparum and $P$. vivax has been reported (Kakar et al., 2010; Khattak et al., 2013). Our findings support previous reports of the high prevalence of malaria in the tribal areas of the Afghanistan-Pakistan border, and increasing prevalence over the last few decades in the Punjab province (Kakar et al., 2010; Khattak et al., 2013). Our results support the reports suggesting that while the majority of the cases of malaria are caused by $P$. vivax, the prevalence of $P$. falciparum has increased during recent years (Khattak et al., 2013). The increased prevalence of $P$. falciparum may be an attribute to antimicrobial resistance; previous studies have shown that the pyrimethamine and chloroquine resistance mutations in $P$. falciparum are present in different cities of Pakistan (Ghanchi et al., 2011). Another explanation for the increased prevalence of $P$. falciparum may be provided by the influx of people and the movement of refugees from areas of Afganistan where the parasite species is common (Howard et al., 2011).

Three structurally distinct types of rDNA $18 \mathrm{~S}$ genotypic variants have been reported in $P$. falciparum and P. vivax laboratory isolates (Li et al., 1994b; McCutchan et al., 1988; Qari et al., 1994; Rogers et al., 1995). The existence of genotypic variants in the field studies has not been described. In the present study, we have identified the two independent gene duplication events that occurred in P. falciparum, leading to the A and S type rDNA18S lineages; the type A lineage being the ancestor of at least two groups (Fig. 6A). In P. vivax, we identified two independent gene duplication events, also leading to the A and S type rDNA18S lineages; the type A lineage being the ancestor of at least three groups (Fig. 6B). In the previous reports, type A was transcribed in erythrocytic schizogony and gametocyte stages consistent with those stages that could have been represented in the present study. In these reports, type $S$ was transcribed in the exoerythrocytic schizogeny stage, while type $O$ was associated with oocyst development only in infected mosquitoes ( $\mathrm{Li}$ et al., 1997). These observed differences in the $18 \mathrm{~S}$ loci of $P$. falciparum and $P$. vivax field samples confirm the presence of genotypic variants. Better understanding is needed of the function of these structurally distinct ribosomes that are active with enhanced transcription during different stages of parasitic development in Plasmodium, with reference to the development of disease control strategies.

In conclusion, we describe for the first time the use of metabarcoded DNA sequencing using an Illumina MiSeq platform to quantify $P$. falciparum and P. vivax, and demonstrate its accuracy on malaria-positive samples. Our results provide a proof of concept for the use of 
the method in disease surveillance, similar to its application in the study of haemoprotozoan parasites of livestock (Chaudhry et al., 2019) and dogs (Huggins et al., 2019). This work was undertaken to explore the possibilities for the application of this high throughput method to determine the dynamics of co-infections, disease biology and epidemiology in Plasmodium parasites, and has applications in monitoring the changes in parasite populations after the emergence and spread of antimicrobial drug resistance (Shaukat et al., 2019).

\section{Acknowledgment}

The study was financially supported by the Roslin Institute uses facilities funded by the Biotechnology and Biological Sciences Research Council (BBSRC). Work at the University of Veterinary and Animal Science Pakistan and Kohat University of Science and Technology Pakistan uses facilities funded by the Higher Education Commission of Pakistan.

\section{Conflict of interest}

None

\section{References}

Agudelo, O., Arango, E., Maestre, A., Carmona-Fonseca, J., 2013. Prevalence of gestational, placental and congenital malaria in north-west Colombia. Malar. J. 12, 1475-2875.

Akinyi Okoth, S., Abdallah, J.F., Ceron, N., Adhin, M.R., Chandrabose, J., Krishnalall, K., Huber, C.S., Goldman, I.F., Macedo de Oliveira, A., Barnwell, J.W., Udhayakumar, V., 2015. Variation in Plasmodium falciparum histidine-rich protein 2 (Pfhrp2) and Plasmodium falciparum histidine-rich protein 3 (Pfhrp3) gene deletions in Guyana and Suriname. PLoS One. 10, e0126805.

Canier, L., Khim, N., Kim, S., Sluydts, V., Heng, S., Dourng, D., Eam, R., Chy, S., Khean, C., Loch, K., Ken, M., Lim, H., Siv, S., Tho, S., Masse-Navette, P., Gryseels, C., Uk, S., Van Roey, K., Grietens, K.P., Sokny, M., Thavrin, B., Chuor, C.M., Deubel, V., Durnez, L., Coosemans, M., Menard, D., 2013. An innovative tool for moving malaria PCR detection of parasite reservoir into the field. Malar. J. 12, 405.

Chaudhry, U., Ali, Q., Rashid, I., Shabbir, M.Z., Ijaz, M., Abbas, M., Evans, M., Ashraf, K., Morrison, I., Morrison, L., Sargison, N.D., 2019. Development of a deep amplicon sequencing method to determine the species composition of piroplasm haemoprotozoa. Ticks Tick Borne Dis. 10, 101276.

Cunha, M.G., Medina, T.S., Oliveira, S.G., Marinho, A.N., Povoa, M.M., Ribeiro-dos-Santos, A.K., 2009. Development of a Polymerase Chain Reaction (PCR) method based on amplification of mitochondrial DNA to detect Plasmodium falciparum and Plasmodium vivax. Acta Trop. 111, $35-38$.

Das, A., Holloway, B., Collins, W.E., Shama, V.P., Ghosh, S.K., Sinha, S., Hasnain, S.E., Talwar, G.P., Lal, A.A., 1995. Species-specific 18S rRNA gene amplification for the detection of $P$. falciparum and P. vivax malaria parasites. Mol. Cell. Probes. 9, 161-165.

Dash, A., Adak, T., Raghavendra, K., Singh, O., 2007. The biology and control of malaria vectors. Curr Sci 92, 1571-1578.

Echeverry, D.F., Deason, N.A., Davidson, J., Makuru, V., Xiao, H., Niedbalski, J., Kern, M., Russell, T.L., Burkot, T.R., Collins, F.H., Lobo, N.F., 2016. Human malaria diagnosis using a single- 
step direct-PCR based on the Plasmodium cytochrome oxidase III gene. Malar. J. 15, 0161185 .

Ghanchi, N.K., Ursing, J., Beg, M.A., Veiga, M.I., Jafri, S., Martensson, A., 2011. Prevalence of resistance associated polymorphisms in Plasmodium falciparum field isolates from southern Pakistan. Malar. J. 10, 18.

Haanshuus, C.G., Mohn, S.C., Morch, K., Langeland, N., Blomberg, B., Hanevik, K., 2013. A novel, single-amplification PCR targeting mitochondrial genome highly sensitive and specific in diagnosing malaria among returned travellers in Bergen, Norway. Malar. J. 12, 1475-2875.

Howard, N., Durrani, N., Sanda, S., Beshir, K., Hallett, R., Rowland, M., 2011. Clinical trial of extended-dose chloroquine for treatment of resistant falciparum malaria among Afghan refugees in Pakistan. Malar. J. 10, 171.

Huggins, L.G., Koehler, A.V., Ng-Nguyen, D., Wilcox, S., Schunack, B., Inpankaew, T., Traub, R.J., 2019. A novel metabarcoding diagnostic tool to explore protozoan haemoparasite diversity in mammals: a proof-of-concept study using canines from the tropics. Scientific Reports 9, 12644.

Huson, D.H., Bryant, D., 2005. Application of phylogenetic networks in evolutionary studies. Mol. Biol. Evol. 23, 254-267.

Kakar, Q., Khan, M.A., Bile, K.M., 2010. Malaria control in Pakistan: new tools at hand but challenging epidemiological realities. East Mediterr Health J. 16, S54-60.

Kearse, M., Moir, R., Wilson, A., Stones-Havas, S., Cheung, M., Sturrock, S., Buxton, S., Cooper, A., Markowitz, S., Duran, C., 2012. Geneious Basic: an integrated and extendable desktop software platform for the organization and analysis of sequence data. Bioinformatics. 28, 1647-1649.

Khattak, A.A., Venkatesan, M., Nadeem, M.F., Satti, H.S., Yaqoob, A., Strauss, K., Khatoon, L., Malik, S.A., Plowe, C.V., 2013. Prevalence and distribution of human Plasmodium infection in Pakistan. Malar. J. 12, 1475-2875.

Kozich, J.J., Westcott, S.L., Baxter, N.T., Highlander, S.K., Schloss, P.D., 2013. Development of a dual-index sequencing strategy and curation pipeline for analyzing amplicon sequence data on the miseq illumina sequencing platform. Appl. Environ. Microbiol. 79, 5112-20.

Lee, P.C., Chong, E.T., Anderios, F., Al Lim, Y., Chew, C.H., Chua, K.H., 2015. Molecular detection of human Plasmodium species in Sabah using PlasmoNex multiplex PCR and hydrolysis probes real-time PCR. Malar. J. 14, 015-0542.

Lefterova, M.I., Budvytiene, I., Sandlund, J., Farnert, A., Banaei, N., 2015. Simple Real-Time PCR and Amplicon Sequencing Method for Identification of Plasmodium Species in Human Whole Blood. J. Clin. Microbiol. 53, 2251-2257.

Li, J., Gutell, R.R., Damberger, S.H., Wirtz, R.A., Kissinger, J.C., Rogers, M.J., Sattabongkot, J., McCutchan, T.F., 1997. Regulation and trafficking of three distinct 18 S ribosomal RNAs during development of the malaria parasite. J. Mol. Biol. 269, 203-213.

Li, J., McConkey, G.A., Rogers, M.J., Waters, A.P., McCutchan, T.R., 1994a. Plasmodium: the developmentally regulated ribosome. Exp. Parasitol. 78, 437-441.

Li, J., Wirtz, R.A., McConkey, G.A., Sattabongkot, J., McCutchan, T.F., 1994. Transition of Plasmodium vivax ribosome types corresponds to sporozoite differentiation in the mosquito. Mol. Biochem. Parasitol. 65, 283-289.

Li, M., Xia, Z., Yan, H., 2014. New type of SSUrDNA sequence was detected from both Plasmodium ovale curtisi and Plasmodium ovale wallikeri samples. Malar. J. 13, 216.

McCutchan, T.F., de la Cruz, V.F., Lal, A.A., Gunderson, J.H., Elwood, H.J., Sogin, M.L., 1988. Primary sequences of two small subunit ribosomal RNA genes from Plasmodium falciparum. Mol. Biochem. Parasitol. 28, 63-68.

Moody, A., 2002. Rapid diagnostic tests for malaria parasites. Clin. Microbiol. Rev. 15, 66-78.

Murillo Solano, C., Akinyi Okoth, S., Abdallah, J.F., Pava, Z., Dorado, E., Incardona, S., Huber, C.S., Macedo de Oliveira, A., Bell, D., Udhayakumar, V., Barnwell, J.W., 2015. Deletion of Plasmodium falciparum histidine-rich protein 2 (pfhrp2) and histidine-rich protein 3 (pfhrp3) genes in Colombian Parasites. PLoS One. 10, e0131576.

Poostchi, M., Silamut, K., Maude, R.J., Jaeger, S., Thoma, G., 2018. Image analysis and machine learning for detecting malaria. Transl Res. 194, 36-55. 
Qari, S.H., Goldman, I.F., Pieniazek, N.J., Collins, W.E., Lal, A.A., 1994. Blood and sporozoite stagespecific small subunit ribosomal RNA-encoding genes of the human malaria parasite Plasmodium vivax. Gene. 150, 43-49.

Rachid Viana, G.M., Akinyi Okoth, S., Silva-Flannery, L., Lima Barbosa, D.R., Macedo de Oliveira, A., Goldman, I.F., Morton, L.C., Huber, C., Anez, A., Dantas Machado, R.L., Aranha Camargo, L.M., Costa Negreiros do Valle, S., Marins Povoa, M., Udhayakumar, V., Barnwell, J.W., 2017. Histidine-rich protein 2 (pfhrp2) and pfhrp3 gene deletions in Plasmodium falciparum isolates from select sites in Brazil and Bolivia. PLoS One. 12, e0171150.

Rakotonirina, H., Barnadas, C., Raherijafy, R., Andrianantenaina, H., Ratsimbasoa, A., Randrianasolo, L., Jahevitra, M., Andriantsoanirina, V., Menard, D., 2008. Accuracy and reliability of malaria diagnostic techniques for guiding febrile outpatient treatment in malaria-endemic countries. Am. J. Trop. Med. Hyg. 78, 217-221.

Rogers, M.J., McConkey, G.A., Li, J., McCutchan, T.F., 1995. The ribosomal DNA loci in Plasmodium falciparum accumulate mutations independently. J. Mol. Biol. 254, 881-891.

Saravu, K., Rishikesh, K., Kamath, A., Shastry, A.B., 2014. Severity in Plasmodium vivax malaria claiming global vigilance and exploration-a tertiary care centre-based cohort study. Malar. J. $13,10.1186$.

Schloss, P.D., Westcott, S.L., Ryabin, T., Hall, J.R., Hartmann, M., Hollister, E.B., Lesniewski, R.A., Oakley, B.B., Parks, D.H., Robinson, C.J., Sahl, J.W., Stres, B., Thallinger, G.G., Van Horn, D.J., Weber, C.F., 2009. Introducing mothur: open-source, platform-independent, communitysupported software for describing and comparing microbial communities. Appl. Environ. Microbiol. 75, 7537-7541.

Scholzen, A., Cooke, B.M., Plebanski, M., 2014. Plasmodium falciparum induces Foxp3hi CD4 T cells independent of surface PfEMP1 expression via small soluble parasite components. Front. Microbiol. 5, 200.

Shaukat, A., Ali, Q., Connelley, T., Khan, M.A.U., Saleem, M.A., Evans, M., Rashid, I., Sargison, N.D., Chaudhry, U., 2019. Selective sweep and phylogenetic models for the emergence and spread of pyrimethamine resistance mutations in Plasmodium vivax. Infect. Genet. Evol. 68, 221-230.

Sinka, M.E., Bangs, M.J., Manguin, S., Rubio-Palis, Y., Chareonviriyaphap, T., Coetzee, M., Mbogo, C.M., Hemingway, J., Patil, A.P., Temperley, W.H., 2012. A global map of dominant malaria vectors. Parasit. Vectors. 5, 69.

Steenkeste, N., Incardona, S., Chy, S., Duval, L., Ekala, M.T., Lim, P., Hewitt, S., Sochantha, T., Socheat, D., Rogier, C., Mercereau-Puijalon, O., Fandeur, T., Ariey, F., 2009. Towards highthroughput molecular detection of Plasmodium: new approaches and molecular markers. Malar. J. 8, 1475-2875.

Tamura, K., Stecher, G., Peterson, D., Filipski, A., Kumar, S., 2013. MEGA6: molecular evolutionary genetics analysis version 6.0. Mol. Biol. Evol. 30, 2725-2729.

William, T., Menon, J., Rajahram, G., Chan, L., Ma, G., Donaldson, S., Khoo, S., Frederick, C., Jelip, J., Anstey, N.M., 2011. Severe Plasmodium knowlesi malaria in a tertiary care hospital, Sabah, Malaysia. Emerg. Infect. Dis. 17, 1248-1255.

Wongsrichanalai, C., Barcus, M.J., Muth, S., Sutamihardja, A., Wernsdorfer, W.H., 2007. A review of malaria diagnostic tools: microscopy and rapid diagnostic test (RDT). Am. J. Trop. Med. Hyg. 77, 119-127.

\section{Figure Legends}

Fig.1. (A) Giemsa-stained blood smears were examined by $1000 \mathrm{x}$ microscopy, showing rings in the Plasmodium positive samples. (B) Immunochromatographic assay for the detection of P. falciparum specific histidine-rich protein 2 (Pf-HRP2) and P. vivax specific lactate dehydrogenase (Pv-LDH). Adequate volumes of the blood samples were dispensed into the sample well ' $\mathrm{S}$ ' of the test cassette. If Pf-HRP2 was bind to the HRP2 gold conjugates 
551 forming a burgundy colored pf band, indicating $P$. falciparum positive test. If $\mathrm{Pv}-\mathrm{LDH}$ was bind to the LDH gold conjugates forming a burgundy colored pv band, indicating $P$. vivax positive test. The absence of any band suggests a negative result and $\mathrm{C}$ is positive control.

Fig. 2. Schematic representation of the sample preparation (A) and the bioinformatics data handling (B) of the metabarcoded sequencing library. (A) In the first-round PCR amplification, overhanging forward and reverse primers were used to amplify the rDNA 18S. The adapter base pairs provide the target sites for the primers used for sequencing and the random nucleotides (0-3Ns) were inserted between the primers and the adapter to offset the reading frame, therefore amplicons prevent the oversaturation of the MiSeq sequencing channels. The second-round PCR amplification was then performed using overhanging barcoded primers bound to the adapter tags to add indices, as well as the P7 and P5 regions required to bind to the MiSeq flow cell. (B) Text files containing rDNA $18 \mathrm{~S}$ sequence data (FASTQ files) were generated from the Illumina MiSeq binary raw data outputs, and data analyses were performed using a bespoke modified pipeline in Mothur v1.39.5 software (Schloss et al., 2009) and Illumina MiSeq standard procedures (Kozich et al., 2013) as described in materials and methods section 2.4.

Fig. 3. The maximum-likelihood tree was obtained from the $P$. falciparum and $P$. vivax rDNA $18 \mathrm{~S}$ region. The sequences were first calculated the number of reference sequences generated from both species (Supplementary Data S1). A total of 34 reference sequences of the rDNA $18 \mathrm{~S}$ locus were identified in $P$. falciparum and 74 reference sequences were identified in $P$. vivax. The reference sequences were aligned on the MUSCLE tool of the Geneious v9.0.1 software. The neighbor-joining algorithm (HKY parameter model) was computed with 1000 bootstrap replicates using MEGA 7 software. Both species were identified with different color shades ( $P$. falciparum in blue and $P$. vivax in brown).

Fig. 4. Validation of the metabarcoding sequencing using three separate mock pools [Mix 1 (P. falciparum), Mix 2 (P. vivax), Mix 3 (P. falciparum and $P$. vivax)] of unknown numbers of parasites from each species. Panel 2A shows that metabarcoded sequencing was used on four replicates of each mock pool to amplify both species as denoted on the X-axis. The Yaxis shows the percentage proportions of each species. Panel 2B shows how the replicates were grouped and averaged based on the amplification. The species are identified with different colours (P. falciparum in blue and P. vivax in brown).

Fig. 5. Immunochromatographic assay (RDT) and the metabarcoding sequencing (Illumina MiSeq) was performed for the detection of $P$. falciparum and $P$. vivax. A total of 365 malariapositive samples were collected in EDTA tube from basic health units in the tribal area of the Pakistan-Afghanistan border and Chughtai Diagnostic Laboratory in the Punjab province of Pakistan. The immunochromatographic assay and the metabarcoded $18 \mathrm{~S}$ rDNA sequencing methods were applied to each sample; the $\mathrm{X}$-axis shows the proportion of each species being estimated and the Y-axis shows the percentage proportions of each species. The species are identified with different colours ( $P$. falciparum in pink and $P$. vivax in light blue).

Fig. 6. Split tree was made for the $P$. falciparum and $P$. vivax $18 \mathrm{~S}$ rDNA sequence data. 134 genotypes were identified in $P$. falciparum and 48 genotypes were identified in $P$. vivax (Supplementary Data S2). The genotypes were aligned on the MUSCLE tool of the Geneious v9.0.1 and the tree was constructed with the UPGMA method in the Jukes-Cantor model of substitution in the SplitsTrees4 software. The appropriate model of nucleotide substitutions was selected by using the jModeltest 13.1.0 program. The pie chart circles in the tree 
601 represent the different $18 \mathrm{~S}$ genotypes containing different colours as follows: (A) $P$. 602 falciparum from the field samples are coloured pink (type A group 1 and 2) and NCBI 603 database sequences are coloured blue (type A group 1 and type S). (B) P. vivax from the field 604 samples are coloured light blue (type A group 1, 2 and 3) and NCBI database sequences are 605 coloured brown (type A group 3 and type $S$ ). 


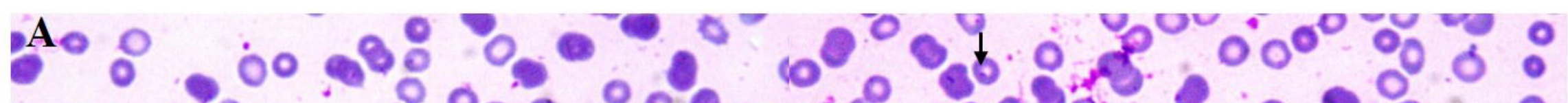

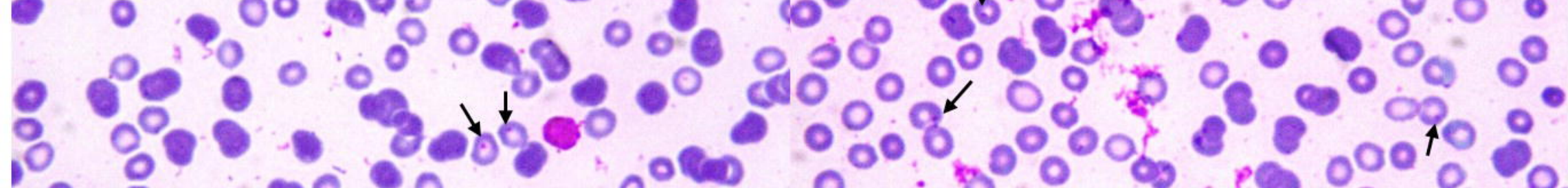
i० o $000 \%$

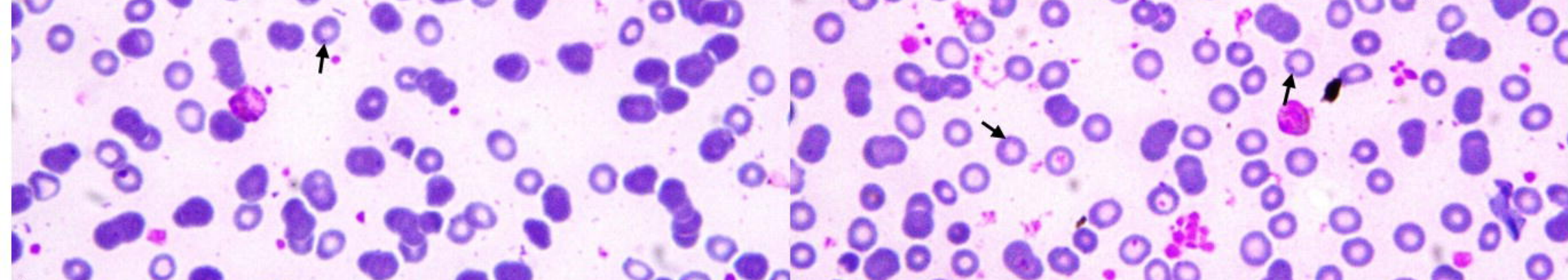

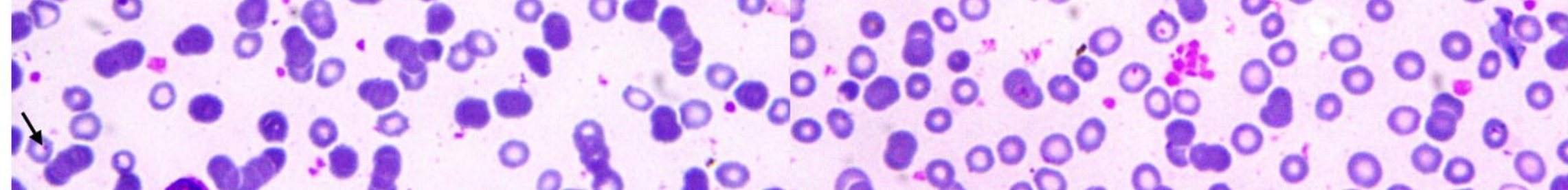

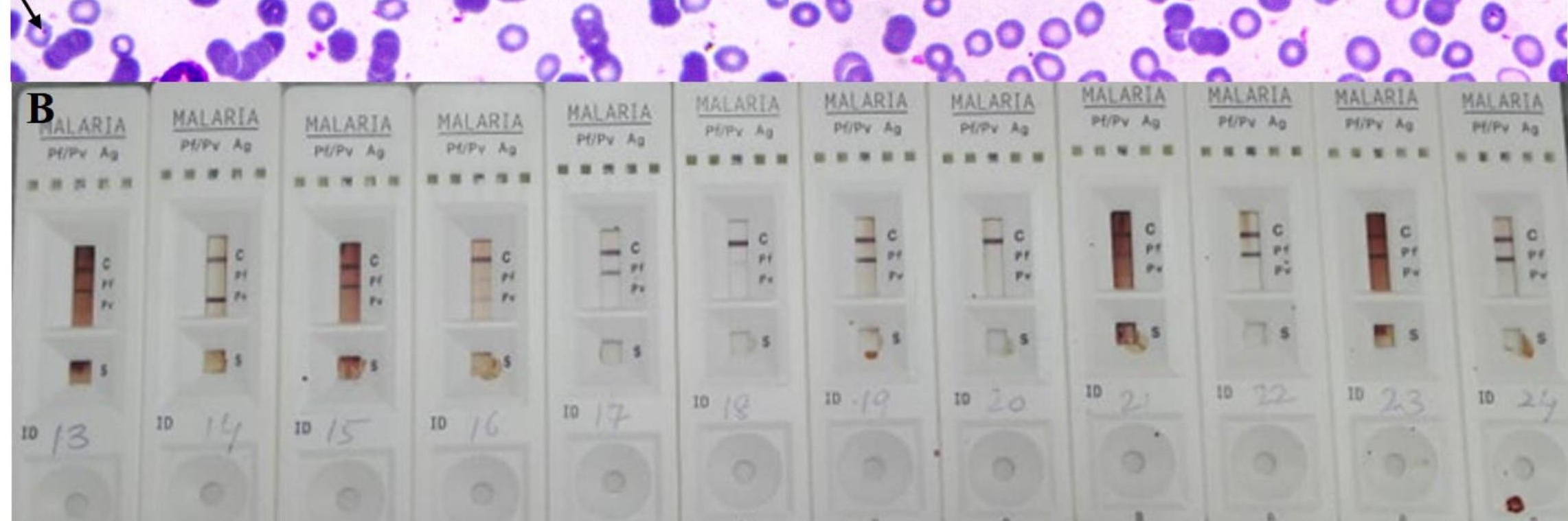


(A) Sample preparation

\section{Adapter PCR}

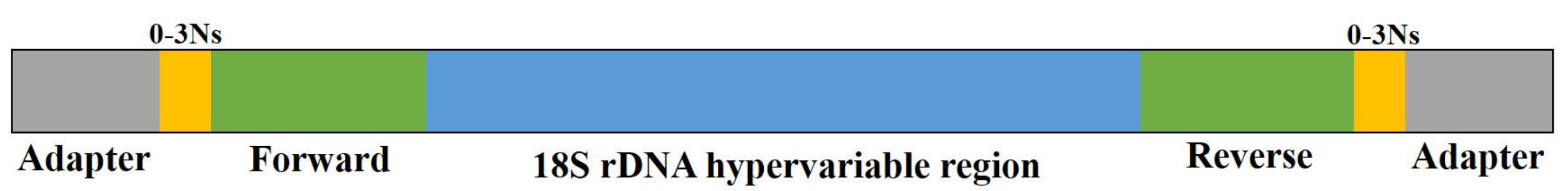

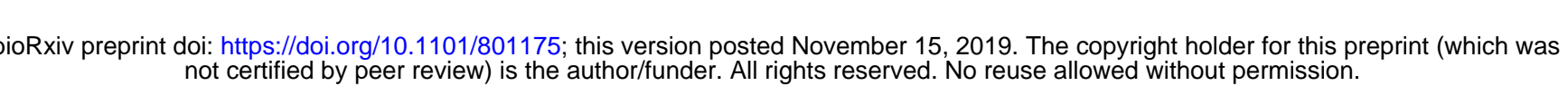

\section{Barcoded PCR}

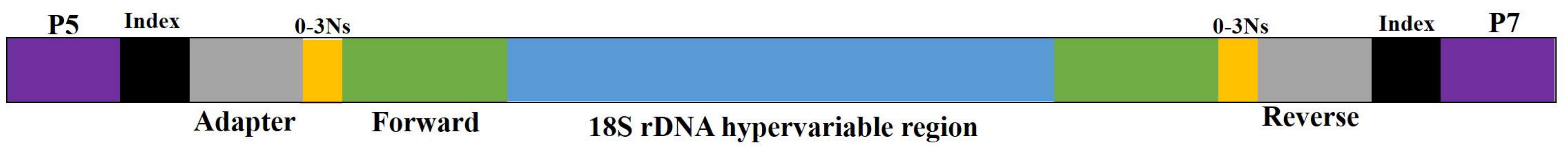

\section{$\underline{\text { 3. Pooling of all samples }}$}

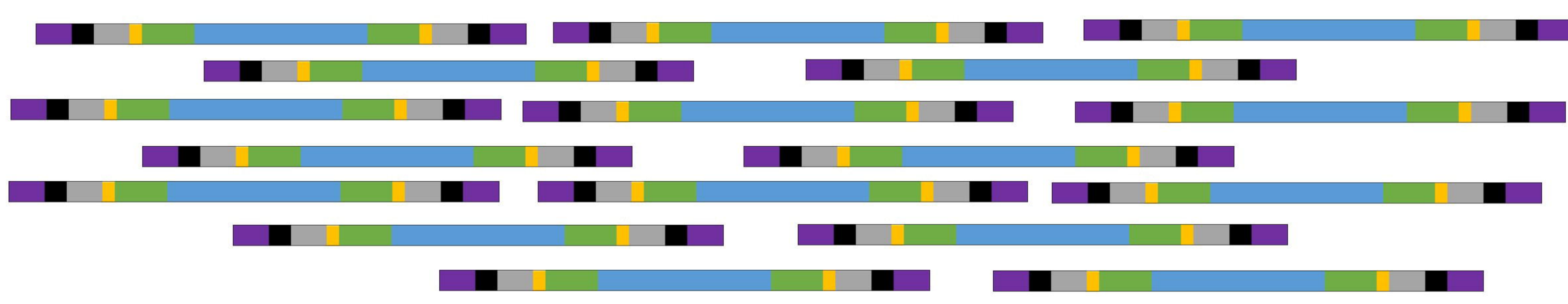

4. Sequencing each sample on Illumina MiSeq platform

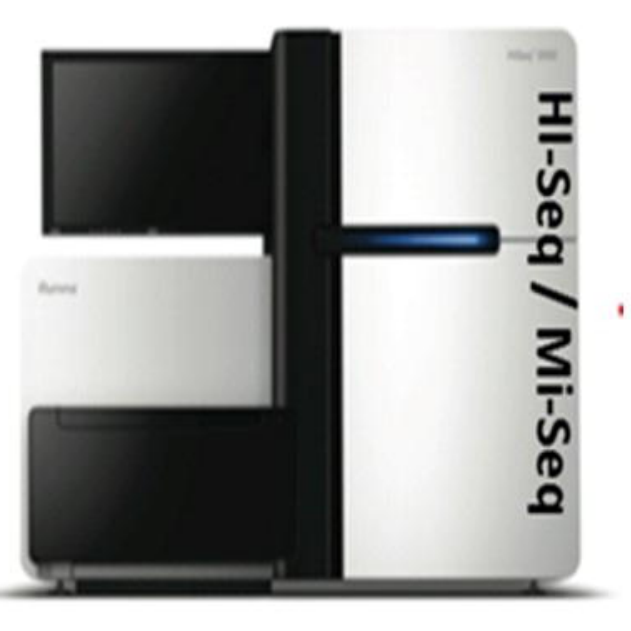

\section{(B) Bioinformatics data handling} $\underline{\text { Step } 1}$

Raw paired-ends reads were analysed (make.contigs' command)

Remove any ambiguous bases (screen.seqs command)

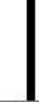

Align dataset with a rDNA 18 S reference sequence library

(align.seqs command)

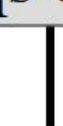

Confirm the filtered sequences overlap the same region of the rDNA $18 \mathrm{~S}$ reference sequence library (screen.seqs command) I

Classify the sequences into either of the two species (P. falciparum and P. vivax) (classify.seqs command)

$\underline{\text { Step } 2}$

Count list of the consensus sequences for each parasite samples based on the total numbers of filtered sequences (unique.seqs command)

Count list was used to create the FASTQ files of the consensus sequences of each sample (split.groups command) 


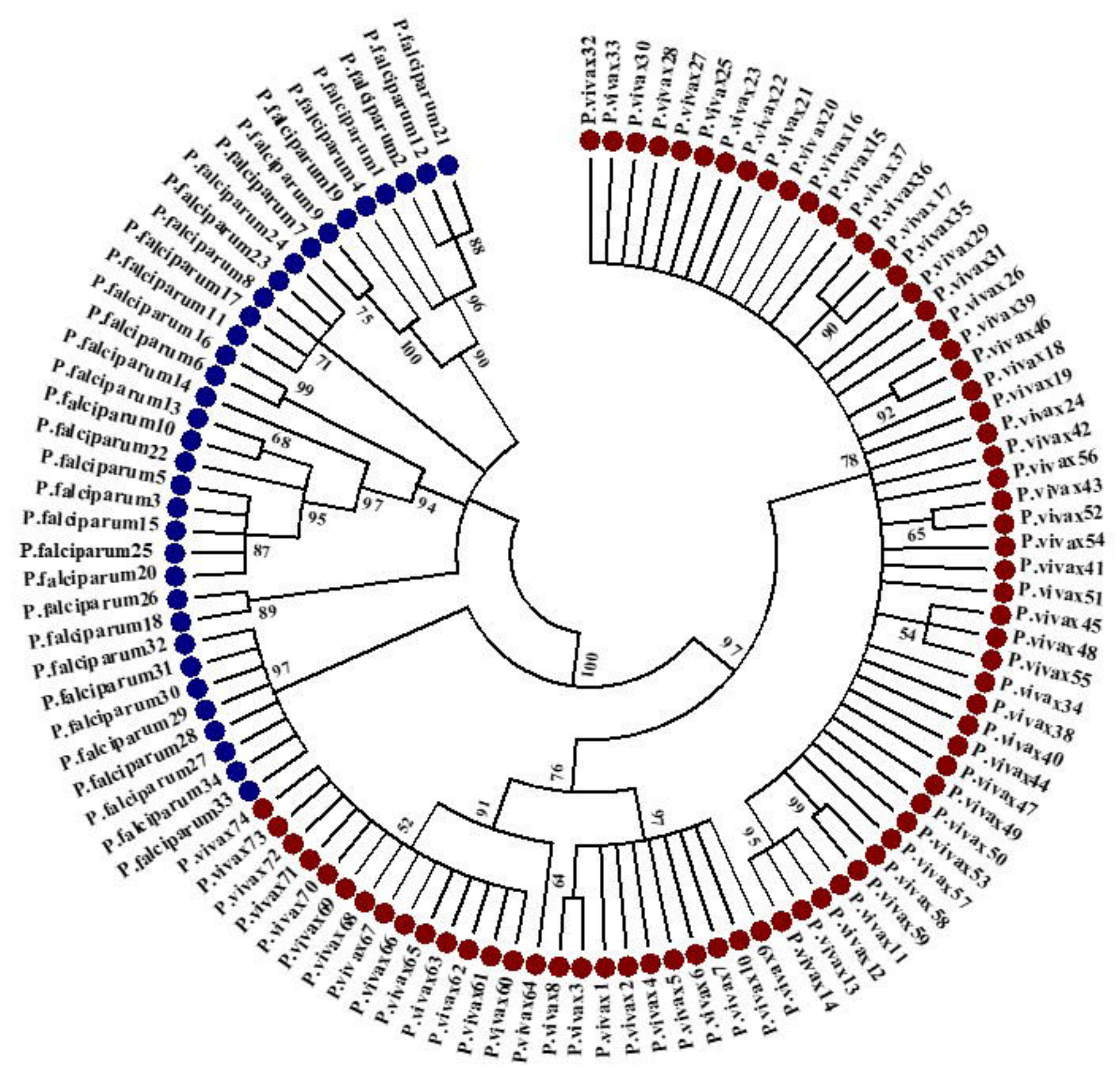


(A) Type A (group 2)
(B)

\section{Clade I}

\section{Clade I}

Type S
$0 \% 8$

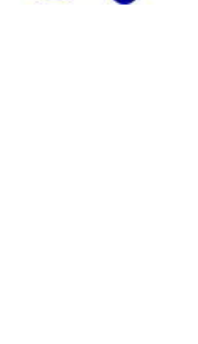

Clade II

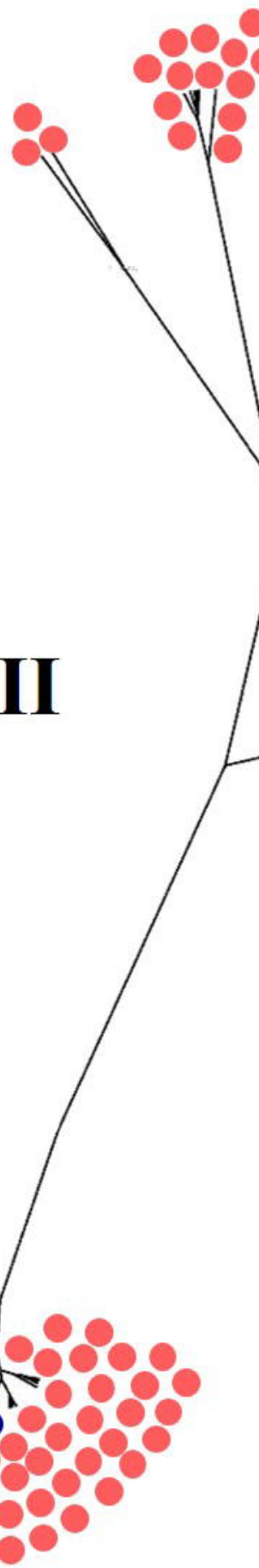

Type A (group 1)

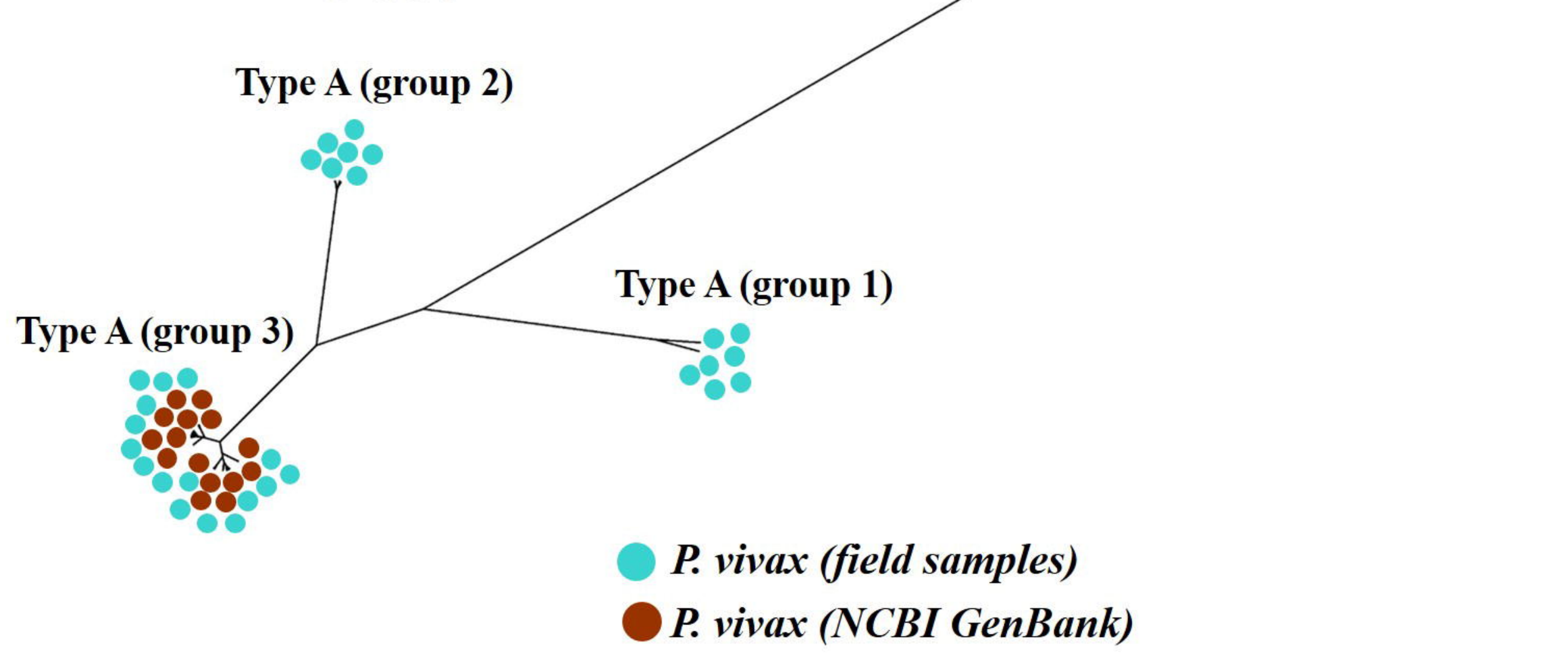

